\title{
The n3He experiment: Hadronic parity violation in cold neutron capture on $3 \mathrm{He}$
}

Michael Gericke*University of Manitoba

E-mail: moerickedphysics.umanitoba.ca

S. Baeßler (University of Virginia), L. Barrón-Palos (Universidad Nacional Autónoma de México), N. Birge (University of Tennessee), J.D. Bowman (Oak Ridge National Laboratory), J.R. Calarco (University of New Hampshire), T.V. Cianciolo (Oak Ridge National Laboratory), C. Coppola (University of Tennessee), C. Crawford (University of Kentucky), J. Favela (Universidad Nacional Autónoma de México), N. Fomin (University of Tennessee), I. Garishvili (Oak Ridge National Laboratory), C. Gautham (University of Tennessee), G.L. Greene (University of Tennessee), V. Gudkov (University of South Carolina), J. Hamblen (University of Tennessee at Chattanooga), C. Hayes (University of Tennessee), L. Kabir (University of Kentucky), S. Kucuker (University of Tennessee), J. Martin (University of Winnipeg), M. McCrea (University of Manitoba), P. Mueller (Oak Ridge National Laboratory), I. Novikov (University of Western Kentucky), C. Olguin (University of Manitoba), S.I. Penttilä (Oak Ridge National Laboratory), E. Plemons (University of Tennessee), M. Scott (University of Tennessee), P.-N. Seo (Duke University), Y-H. Song (University of South Carolina), A. Sprow (University of Kentucky), M. Schindler (University of South Carolina), J. Thomison (Oak Ridge National Laboratory), M. Viviani (INFN-Pisa), J. Watts (University of Tennessee at Chattanooga)

\footnotetext{
The $\mathrm{n} 3 \mathrm{He}$ experiment aims to measure the parity violating asymmetry in the direction of proton emission in the reaction $\vec{n}+{ }^{3} \mathrm{He} \rightarrow{ }^{3} \mathrm{H}+\mathrm{p}$, using the capture of polarized cold neutrons in an unpolarized gaseous ${ }^{3} \mathrm{He}$ target. Using effective field theory based calculations, the size of the asymmetry is estimated to be in the range of $(-10 \rightarrow 2) \times 10^{-8}$, depending on the framework in which the calculations are done, and our goal measurement accuracy is $2 \times 10^{-8}$. The asymmetry is a result of the low energy weak interaction between nucleons and its measurement will provide a benchmark for modern effective field theory calculations in the isospin $=0$ channel. The experiment uses a ${ }^{3} \mathrm{He}$ multiwire ionization chamber as the combined target and detector, operated in current mode. The $n 3 H e$ experiment was installed and commissioned in December 2014 on the Fundamental Neutron Physics Beamline at the Spallation Neutron Source at Oak Ridge National Laboratory. The experiment is currently taking production data. In this paper, we provide an overview of the experiment and will show a few preliminary results of the data collected to date.
}

The 8th International Workshop on Chiral Dynamics

29 June 2015 - 03 July 2015

Pisa, Italy 


\section{Introduction}

The hadronic weak interaction at low energy can be described by 5 independent amplitudes labeled by the isospin change. In the meson exchange picture the weak NN interaction is modeled by the weak coupling of the meson at one vertex and the strong coupling of the meson at the other vertex, leading to a description in terms 5 coupling constants corresponding to different meson-isospin combinations [四]. In more modern effective field theory (EFT) based approaches, the interaction is modeled by writing down a Lagrangian in terms of nucleon degrees of freedom, which is consistent with the symmetries of QCD and then performing an expansion in terms of momentum transfer $\left(q / \Lambda_{\chi}\right)$ [回, 目, 目, [, 团]. To lowest order (for cold neutrons the momentum transfer is essentially zero), one obtains pion-less effective field theory, in which the resulting PV NN potential again contains 5 low energy coupling constants. However, in reality, the relative size of the NN weak amplitudes in different spin and isospin channels might not be determined entirely by simple symmetry arguments. This is because they are sensitive to the short-range correlations between quarks in the nucleon and to strong NN correlations. The NN weak amplitudes are therefore a good probe of the poorly understood confinement and chiral symmetry breaking dynamics of QCD. New efforts to calculate parity violating NN observables, starting from ( $\chi P T$ based) EFT are being undertaken, but experimental input is needed to relate them to modern NN potentials. A new calculation of the weak nucleon coupling amplitudes on the lattice has recently been completed as well [ [] . The experimental challenge lies in finding feasible few nucleon experiments which can measure the various observables, with high enough accuracy, and together over-constrain the theoretical couplings. This has been made possible in the past few years, due to the availability of new, high intensity neutron facilities, such as the SNS. Over the past 8 years, we have made significant contributions to this field, due to our work on the two highest precision few nucleon measurements to date.

The $n 3 H e$ experiment is a new test of the hadronic weak interaction, measuring the parity violating asymmetry $A_{P V}$ in the correlation between the polarization of incoming cold neutrons $\left(\vec{\sigma}_{n}\right)$ and the outgoing momentum of protons $\left(\vec{k}_{p}\right)$ in the reaction $\vec{n}+{ }^{3} \mathrm{He} \rightarrow p+T+765 \mathrm{keV}$. The size of the asymmetry has been calculated both based on the DDH formalism $\left(A_{P V}=(-9.4 \rightarrow 2.5) \times\right.$

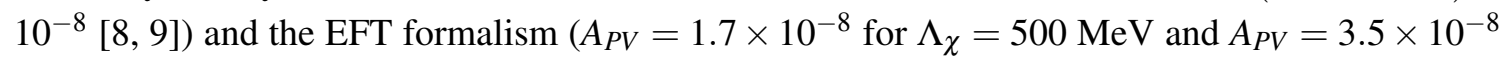
for $\Lambda_{\chi}=600 \mathrm{MeV}$ [四]). The $n 3 H e$ experiment is an important PV measurement because it is an independent probe of the hadronic weak couplings. Together with NPDGamma [ए]], neutron

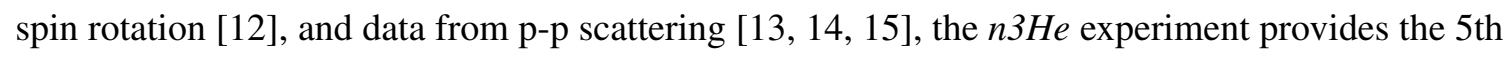
measurement in a few body system with straight forward theoretical interpretation of the results, constraining the full set of weak nucleon-nucleon couplings.

\section{The Experiment}

The experimental setup is shown in Fig. $\mathbb{~}$. The major pieces of hardware are the RF spin rotator, 200 channels of DAQ ADC modules, and the combined ${ }^{3} \mathrm{He}$ target-detector ion chamber. The neutron beam is polarized by a remnant super-mirror polarizer. The initial polarization is transverse and vertically pointing up. The spin of the neutron is then rotated to spin down for every

\footnotetext{
* Speaker.
} 
other pulse, using a spin rotator. The polarized neutrons then capture in a thick ${ }^{3} \mathrm{He}$ ion chamber which is mostly sensitive to the signal from the emitted protons, as explained further below.
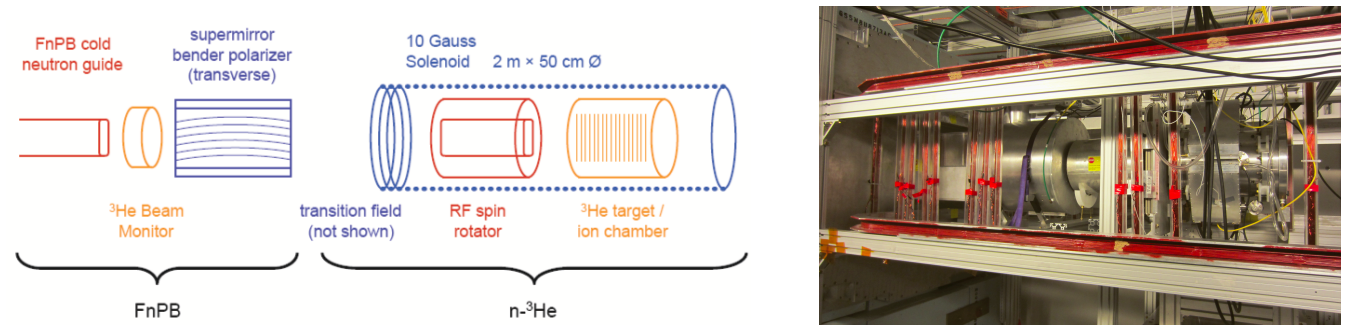

Figure 1: Left: Setup schematic for the $n 3 H e$ experiment. Right: The installed experiment.

The measured asymmetry has both parity violating (up-down) and parity conserving (leftright) contributions:

$$
A_{m s r}=\varepsilon P_{n}\left(A_{P V} G_{P V}+A_{P C} G_{P C}\right) .
$$

Where $\varepsilon$ is the spin rotator efficiency, $P_{n}$ is the neutron beam polarization, and $G_{P V}=\left\langle\cos \theta_{\sigma, k_{p}}\right\rangle$ and $G_{P C}=\left\langle\sin \theta_{\sigma, k_{p}}\right\rangle$ are geometry factors: The angular dependence of the parity violating and parity conserving asymmetry, averaged over the neutron capture distribution in the target, as well as over the size of a given detector element (see below), and over the neutron wavelength.

\subsection{The Target-Detector Chamber}

The ${ }^{3} \mathrm{He}$ target consists of a cylindrical chamber with an inside length of $33.83 \mathrm{~cm}$ and an inside diameter of $25.4 \mathrm{~cm}$. The chamber is made entirely from aluminum, with the exception of the stainless steel knife edges on the conflat flanges for the chamber windows and feedthroughs. The aluminum windows are $1 \mathrm{~mm}$ thick. The chamber is filled with ${ }^{3} \mathrm{He}$, at 0.476 atm $\left(\right.$ at $21^{\circ} \mathrm{C}$ ), without any other gas. The only signal produced in the chamber is due to ionization of the ${ }^{3} \mathrm{He}$ gas, by the protons and tritons moving through the chamber after neutron capture, as well as from electrons due to beta decay after neutron capture on other materials. The target contains 33 wire frames, 16 signal frames with 9 wires each and 17 high voltage (HV) frames with 8 wires each, dividing the target chamber into 144 "cells" that each function as an individual detector (see Figs. $\square$ and (3).

The HV wire bias is -350 Volts, so that the operating condition of the target-detector corresponds to that of an ionization chamber, in which the charge collected on the signal wires is proportional to the energy deposited in each cell. The wire frames are made from macor, a glass ceramic with a low neutron capture cross-section, and the wires are made from $0.5 \mathrm{~mm}$ copper wire. The separation between signal planes and signal wires within a plane is about $1.9 \mathrm{~cm}$. The low gas density, the relatively thick wires, and the low bias voltage guarantee that the ionization chamber signals are linearly dependent on the energy deposited by the protons and the tritons, which ensures that the measured asymmetry is independent of beam intensity and gas gain. Simulations done with the Garfield++ software show that almost all of the charge liberated within a given cell (as they are 
defined in Fig. [1]) is collected at the cathode wire in the center of that cell. The chamber signals are read out in integration mode, using a separate transimpedance amplifier for each wire, which integrates the current in each wire over a time period set by the amplifier bandwidth. The amplified signal is then sampled by ADCs.
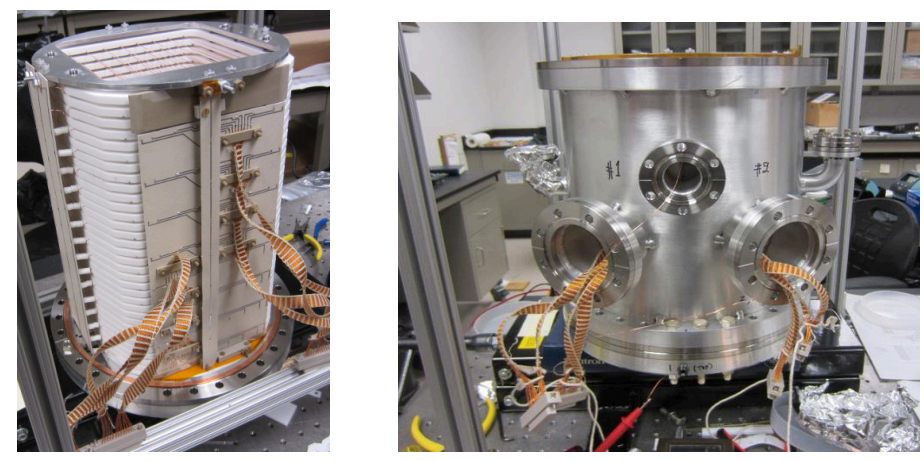

Figure 2: Left: The wire frame stack. Right: The target-detector chamber.

The design of the chamber and the material choices produce negligible background. Possible backgrounds include $\gamma$ - rays from neutron capture in parts along the beam line (in particular the polarizer) and from neutron capture on materials other than ${ }^{3} \mathrm{He}$, in the target itself, as well as $\beta$-decay electrons from neutron capture in the target. The interaction probability for gamma-rays in ${ }^{3} \mathrm{He}$ is smaller by a factor of $\simeq 10^{-3}$, compared to that for cold neutrons and the total neutron scattering cross-section is smaller than the absorption cross-section by a factor of $\simeq 10^{-4}$. This, together with the small amounts of solid material used in the construction of the chamber (e.g. the thin windows), and the size of the chamber as compared to the beam profile, means that background from neutron capture on materials other than on ${ }^{3} \mathrm{He}$ is negligible. Note that the transverse size of the target is larger than the collimated beam profile $(10 \mathrm{~cm}$ horizontal and $8.2 \mathrm{~cm}$ vertical $)$ by about a factor of 3.

The operating principle behind the chamber relies on the fact that the emitted protons carry most of the kinetic energy $\left(k_{T}=k_{p} / 3\right)$ and have a longer range in the target than the tritons and that the protons deposit most of their energy toward the end of their track, while the tritons deposit more of their energy at the beginning of the track (see Fig. T right). Both the proton and triton signal carry the asymmetry in eqn. 2.2 , but the asymmetry is linearly dependent on the outgoing momentum [ [8], so that $A_{T} \simeq-A_{p} / 3$. Further more, due to the smaller range and energy deposition of the tritons, there is a further reduction in the sensitivity to the asymmetry carried by the triton signal. Nevertheless, the back to back emission of the proton and triton produces a partial cancellation of the asymmetry signal, which leads to a statistical degradation to roughly a factor of 3 above neutron counting statistics.

Since neutrons are polarized along the vertical axis, the target chamber is oriented such that the wires are horizontal. This is the default orientation of the chamber for the parity violating measurement (see Fig. [1]). For the parity conserving measurement, the chamber is rotated by $90^{\circ}$ around the beam axis, such that the wires are vertical. Regardless of which way the chamber is rotated, we always form the measured asymmetry from the yield in a pair of wire cells that 

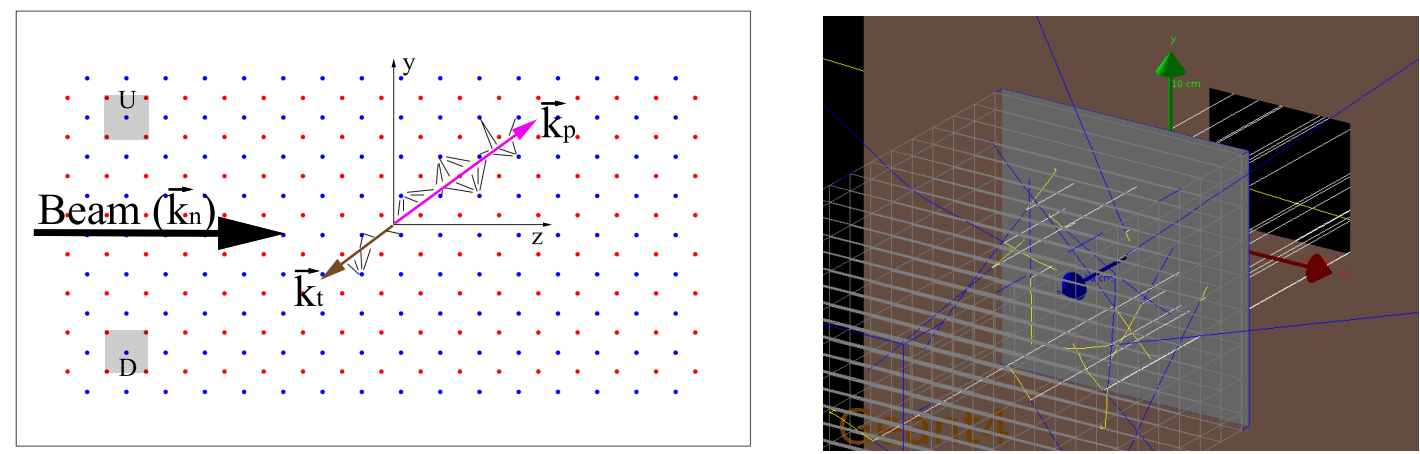

Figure 3: Left: The wire layout of the chamber with an example proton and triton track. The high voltage wires are red and the signal wires are blue. The coordinate system of the experiment is defined, such that z-axis points along the beam direction and the $y$-axis points vertically up, while the $\mathrm{x}$-axis is horizontal and points into page, in this picture. Thus, the wires are oriented along the horizontal. This is the default orientation for the parity violating measurement. The shaded regions indicate two cells of the chamber, defined by $4 \mathrm{HV}$ wires, one at each corner, and one grounded signal wire at the center. The two cells are rotated $180^{\circ}$ with respect to each other, around the beam axis, and form an up-down pair, with respect to the vertical and the un-rotated neutron polarization. Right: Simulation setup with housing removed, showing the wire cell layout in three dimensions. The cells are about $16 \mathrm{~cm}$ long, in the horizontal direction and $1.9 \times 1.9 \mathrm{~cm}^{2}$ along the beam direction and the vertical direction. Neutrons are shown in white, protons in blue, and tritons in yellow.

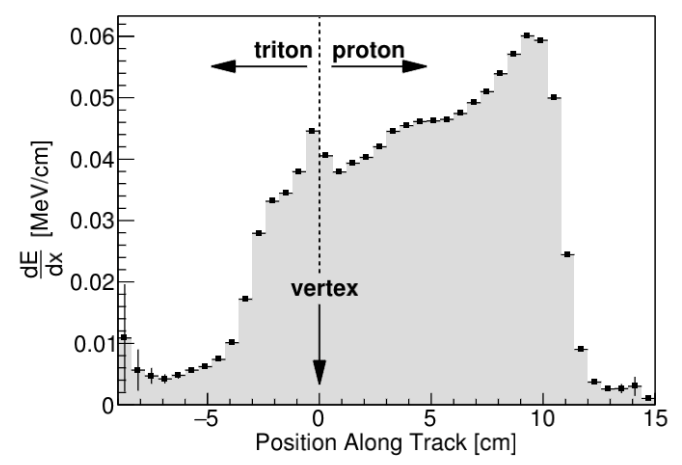

Figure 4: Simulated energy deposition as a function of position along the track for protons and tritons, shown here emitted back to back.

are located on opposite sides of the beam centroid, at equal distances from the beam centroid, or equivalently, from the center z-axis of the target. Figure B left shows an example of such a cell pair, labeled U/D, for up-down in the parity violating measurement. For the parity conserving measurement, the cells would become the left-right pair. The measured asymmetry is formed for each cell pair, according to

$$
A_{m s r}=\frac{\frac{Y_{U}^{+}}{Y_{D}^{+}}-\frac{Y_{U}^{-}}{Y_{D}^{-}}}{\frac{Y_{U}^{+}}{Y_{D}^{+}}+\frac{Y_{U}^{-}}{Y_{D}^{-}}} .
$$


Where the \pm signs stand for the neutron spin polarization with respect to the vertical axis (up/down). This way of forming the asymmetry cancels out both beam fluctuations and gain differences between the wires. For each cell pair, this measured asymmetry is related to the physics asymmetries in eqn. 2.2, with the corresponding geometry factors for that cell pair. Since the cell pairs used in the asymmetry calculation are on opposite sides of the beam axis through the target, and relatively far apart, the signals from these two cells are uncorrelated. However, a given cell pair asymmetry may be correlated to that of a neighboring cell pair, because the proton (or triton) track connects the two cell pairs. This correlation has to be taken into account, when combining the cell pairs into a final asymmetry.

\section{First Data Samples}

The experiment has been collecting production data since February 2015. Here we show a few examples of the data that has been collected. Figure \$ shows two typical time of flight spectra seen in single wires, one at the front of the chamber and one at the back of the chamber (in this case, both wires were located at the vertical center of the chamber). The data is collected in 49 time bins, each of which is $0.32 \mathrm{~ms}$ wide, but the first 5 time bins are discarded, because they have too much signal from slow neutron leakage from previous pulses. The data analysis is based on the signal between about $3.4 \AA$ and $6.5 \AA$. The analysis can be done for each wavelength separately or by integrating the spectrum in each wire over the time of flight range in each pulse.
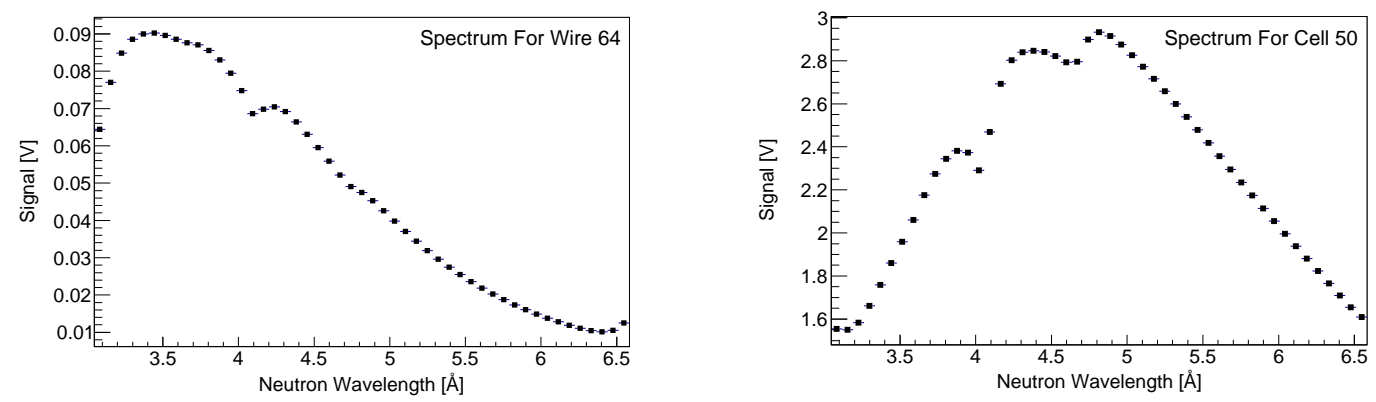

Figure 5: Typical neutron time of flight spectrum from two single wires, one at the front of the chamber (wire 50) and one from the back of the chamber (wire 64). The measured spectra are separated into 49 time bins, each $0.32 \mathrm{~ms}$ wide. Only the signal between $3.4 \AA$ and $6.5 \AA$ is used in the analysis.

Figure 6 shows the signal magnitude for all wires (cells) in the chamber, integrated over the time of flight spectrum and all pulses in a single run. Examples of wire pairs used for asymmetry calculations include frame 2, wires 2 and 8, wires 3 and 7, wires 4 and 6, and similarly for the other frames (except frame 1). Figure $\square$ shows two examples of asymmetry data. Shown on the left are parity conserving asymmetries for a subset of the wire pairs. The left-right parity conserving asymmetry, while being important in its own right, as a benchmark for strong interaction NN models, is an important reality check for the experiment, since it easily measured. The asymmetry is large and the structure due to the geometry factors are clearly visible, serving as a benchmark 
for our calculation of the geometry factors. After the geometry factors are applied, the asymmetry data should smooth out as a function of cell number (with the exception of statistical variations). The right side in Fig. $\square$ shows an example of parity violating asymmetry data. In this case the asymmetry was calculated for each wire, using consecutive neutron pulses with opposite spin. This method will not be used for the calculation of the final asymmetry, since it does not cancel out possible neutron beam fluctuations. It does however serve as a cross-check.

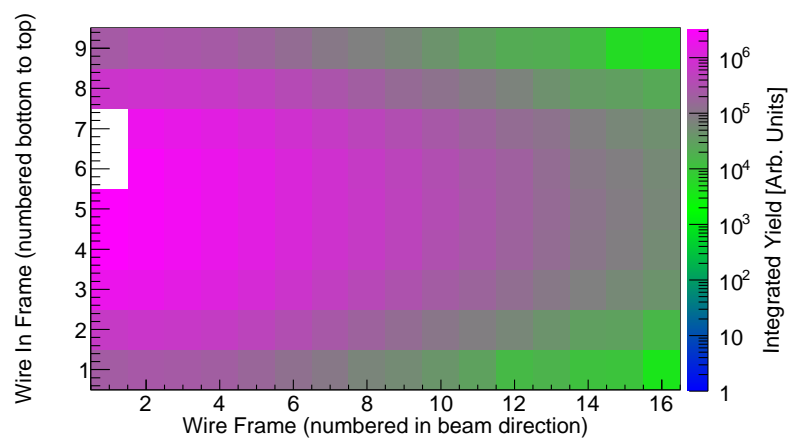

Figure 6: Signal magnitude for each cell in the chamber, integrated over the time of flight spectrum and all pulses in a run (about 25000 in this case). Wires 6 and 7 in frame 1 are not used.
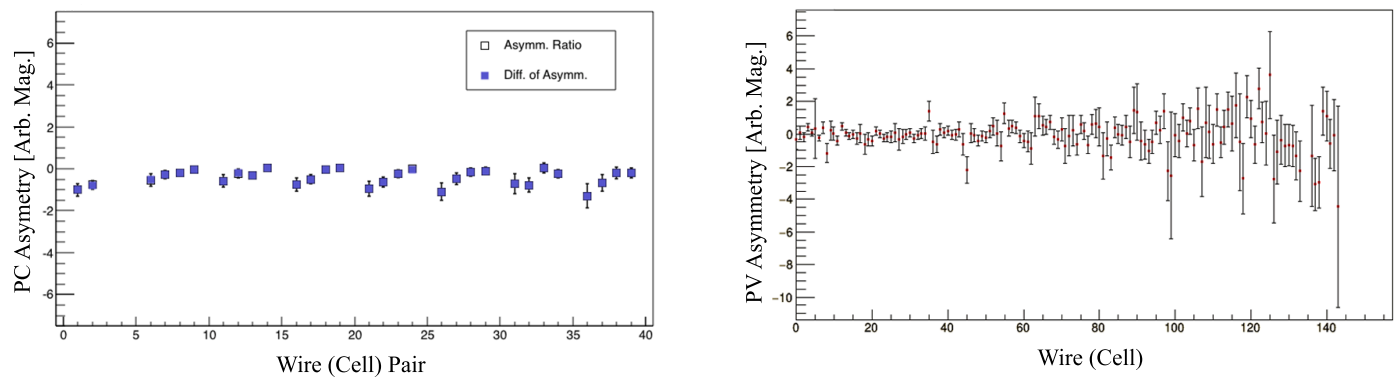

Figure 7: Examples of asymmetry data, for illustrative purposes only. Left: Parity conserving wire pair asymmetries for a subset of the wire pairs. The asymmetry is large and the structure due to the geometry factors is clearly visible. Right: Parity violating single wire asymmetries, calculated from consecutive pulses with opposite neutron spin, but from the same wire.

\section{Conclusion and Outlook}

The $n 3 H e$ experiment has been constructed to produce a precise measurement of the parity violating directional asymmetry in the number of outgoing protons, after neutron capture on ${ }^{3} \mathrm{He}$. The asymmetry is related to the weak nucleon-nucleon coupling amplitudes calculated using modern effective field theory calculations and lattice QCD. The experiment was installed and commissioned in 2014 and early 2015 and has been taking production data since February 2015. The Conclusion of data production is scheduled for the end of 2015. With the available data we expect to reach our goal of $2 \times 10^{-8}$. 


\section{References}

[1] B. Desplanques, J. F. Donoghue and B. R. Holstein, Annals of Physics, 124, 449 (1980)

[2] M.R. Schindler and R.P. Springer, Prog. Part. Nucl. Phys. 72, 1 (2013)

[3] W.C. Haxton and B.R. Holstein, Progr. in Part. Nucl. Phys. 71, 185 (2013)

[4] M.J. Ramsey-Musolf and S.A. Page, Ann. Rev. Nucl. Part. Sci 56, 2 (2006)

[5] J. Erler and M.J. Ramsey-Musolf, Prog. Part. Nucl. Phys. 54, 351 (2005)

[6] C.-P. Liu, Phys. Rev. C 75, 065501 (2007)

[7] J. Wasem, Phys. Rev. C 85, 022501(R) (2012)

[8] M. Viviani, R. Schiavilla, L. Girlanda, A. Kievsky, and L. E. Marcucci, Phys. Rev. C. 82, 044001 (2010)

[9] L. Girlanda et al., Phys. Rev. Lett. 105, 232502 (2010)

[10] M. Viviani, et al., Phys. Rev. C 89, 064004 (2014)

[11] M. T. Gericke et al., Phys. Rev. C 83, 015505 (2011)

[12] W.M. Snow et al., Phys. Rev. C 83, 022501(R)

[13] P.D. Evershiem et al. Phys. Lett. 256 (1991) 11

[14] S. Kistryn et al., Phys. Lett. 58 (1987) 1616

[15] R. Balzer et al., Phys. Rev. C. 30 (1984) 1409

[16] J. F. Caviagnac et al., Phys. Lett. 67B , 148 (1977)

[17] M.T. Gericke et al., Phys. Rev. C 74, 065503 (2006)

[18] M.T. Gericke, et al., Nucl. Instr. Meth. A 540 (2005) 328.

[19] J.F. Donoghue, E. Golowich, and B. Holstein, Phys. Rep. 31 (1986) 319

[20] S. L. Zhu et al., Nuclear Physics A 748, 435 (2005) 